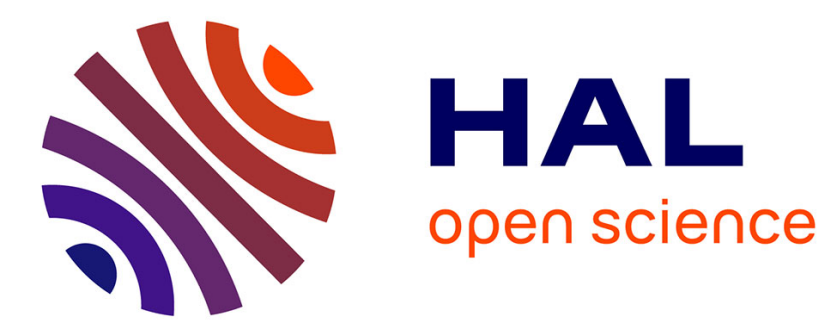

\title{
Does tilt/translation ratio affect perception of deceleration in driving simulators?
}

Anca M. Stratulat, Vincent Roussarie, Jean-Louis Vercher, Christophe Bourdin

\section{- To cite this version:}

Anca M. Stratulat, Vincent Roussarie, Jean-Louis Vercher, Christophe Bourdin. Does tilt/translation ratio affect perception of deceleration in driving simulators?. Journal of Vestibular Research, 2011, 21 (3), pp.127-139. 10.3233/VES-2011-0399 . hal-01436025

\section{HAL Id: hal-01436025 https://hal.science/hal-01436025}

Submitted on 2 May 2018

HAL is a multi-disciplinary open access archive for the deposit and dissemination of scientific research documents, whether they are published or not. The documents may come from teaching and research institutions in France or abroad, or from public or private research centers.
L'archive ouverte pluridisciplinaire HAL, est destinée au dépôt et à la diffusion de documents scientifiques de niveau recherche, publiés ou non, émanant des établissements d'enseignement et de recherche français ou étrangers, des laboratoires publics ou privés. 


\title{
Does tilt/translation ratio affect perception of deceleration in driving simulators?
}

\author{
Anca M. Stratulat ${ }^{\mathrm{a}, \mathrm{b}, *}$, Vincent Roussarie ${ }^{\mathrm{b}}$, Jean-Louis Vercher ${ }^{\mathrm{a}}$ and Christophe Bourdin ${ }^{\mathrm{a}}$ \\ a UMR 6233 "Institut des Sciences du Mouvement”, CNRS - Université de la Méditerranée, Faculté des Sciences \\ du Sport, Marseille Cedex, France \\ ${ }^{\mathrm{b}}$ PSA Peugeot Citroën, Centre Technique de Vélizy, Vélizy-Villacoublay Cedex, France
}

Received 24 June 2010

Accepted 15 February 2011

\begin{abstract}
Tilt-coordination is a technique which uses the tilt-translation ambiguity of the vestibular system to simulate linear accelerations on dynamic driving simulators, in combination with real linear accelerations. However, the tilt/translation ratio is chosen empirically. We experimentally determine the most realistic tilt/translation ratio to simulate a given value of deceleration. Under specific conditions of driving simulation, five tilt/translation ratios were applied, with an inverse-proportional quantity of tilt and translation, so that the sum of the two (the proportion of the deceleration simulated by translational motion and the proportion simulated by tilt) was always equal to the same overall value $\left(0.8 \mathrm{~m} / \mathrm{s}^{2}\right)$. We find that different ratios lead to different perceptions, depending on the quantity of tilt and translation. With a higher tilt ratio, the braking is perceived as being stronger than when there is a higher translation ratio and the most realistic tilt/translation ratio found is neither pure tilt, nor pure translation, but $35 / 65 \%$ tilt/translation. The way these different ratios are perceived during braking is discussed from vestibular and non-vestibular points of view.
\end{abstract}

Keywords: Self-motion perception, vestibular inputs, tilt coordination, braking

\section{Introduction}

How we perceive self-motion from different sensory inputs (namely visual, vestibular and somesthesic) is still not completely understood, in spite of extensive research in neuroscience, psychology and motor control. A comprehensive, predictive theory of multisensory integration is needed, in particular to build more efficient motion simulators for industry.

Over the past two decades, driving simulation has undergone major expansion, not only because of technological advances, but also due to increased interest from car manufacturers and scientists. Driving simula-

*Corresponding author: Anca M. Stratulat, UMR 6233 "Institut des Sciences du Mouvement”, CNRS - Université de la Méditerranée, Faculté des Sciences du Sport, 163, avenue de Luminy - CP 910, 13288 Marseille cedex 09, France. Tel.: +33 (0) 6780556 43; Fax: +33 (0) 4911722 04; E-mail: anca.stratulat@ gmail.com. tors are a useful tool in the vehicle development process (lower costs and time gains during prototyping phase) and for safety (to avoid exposing people to danger in car-driving experiments). Simulators are also powerful tools for a better understanding of self-motion perception and the process of integration of multiple sensory cues (visual, vestibular, auditory, tactile etc). The present study, developed on the dynamic driving simulator of PSA Peugeot-Citroën, investigates the perception of braking during car driving in order to improve dynamic simulations.

Visual information has long been known to play a significant role in the perception of self-motion in the environment. The first driving simulators were motionless, and the quality of the simulation was only determined by the quality of the visual scenes. This type of perception is based on the displacement of optic flow patterns on the retina, offering information about direction of motion, velocity $[17,18]$, time-to-contact 
or relative distances [7]. If the observer is stationary and the visual surroundings are moving, a vection often takes place (a vection is an illusion of motion [29]). All driving simulators are based on this kind of illusion, but recent studies show that visual information alone is not sufficient to provide a realistic simulation [19]. Real movement, which also stimulates vestibular and somesthesic sensors, needs to be added to improve the simulations. Evidence shows that non-visual sensory inputs play a role in all everyday life situations, including postural control, spatial orientation and navigation, walking, sports or driving a vehicle $[5,15,20,21,23,24$, 30,35]. Therefore, even in simulators, vestibular and somesthesic cues appear to be essential to induce a realistic perception of self-motion. Moreover, lack of motion, as well as delayed onset of vection (considered to be in the range of a few seconds $[4,11]$ ), is considered to be a major cause of simulator sickness [32]. For all these reasons, many of the driving simulators used in industry rapidly evolved from static to dynamic architectures. The addition of six degrees-of-freedom hexapods and/or X-Y platforms has been found to facilitate a more realistic simulation. However, the mechanical limitations of dynamic simulators do not allow simulation of all types of driving situations, such as linear displacements with high and/or long-lasting accelerations (take-offs, emergency braking) or tight turns. To go beyond these bounds, two solutions are used by the car manufacturers: 1/ changing the "gain" of the simulator or $2 /$ using the "washout" algorithm. The gain represents the ratio between two values: the value simulated visually and the value simulated by the motion of the simulator. For example, a turn can be represented $100 \%$ on the visual display while only $60 \%$ of the movement is produced by the motion platform, meaning that a gain of 0.6 is used. Interestingly, this specific value (0.6) of the gain has been found to give the most believable simulations $[13,14]$. The "washout" algorithm was introduced first in aeronautics, by Nahon and Reid in 1990 [31], for flight simulations. As linear accelerations during flights are quite high and take place over a long period of time, it is impossible to simulate them by pure translation alone. The "washout" algorithm allows high accelerations to be simulated by combining cell tilt and translation. This algorithm is based on a technique called "tilt-coordination". It supposes the inclination of the simulator in order to orient the driver's head relative to gravity in a way similar to how the gravito-inertial acceleration (GIA) is oriented in the real vehicle during acceleration. The real tilt of the simulator is produced in coordination with the visual representation of the motion [19]: either the display is attached to the cell and thus tilted in the same way, or the visual scene is tilted in the same direction as the cell to limit visual perception of the tilt. This technique is based on the tilt-translation ambiguity given by the vestibular system in the dark ${ }^{1}[1]$. In the absence of vision, this ambiguity leads to perceptually equivalent situations (given that the canals are not responding to the tilt, which is common with dynamics below the thresholds of these organs), as described by Holly and McCollum [22]: a slow rotation of the head, kept under the threshold of inclination of the semicircular canals (6 degrees [9]) will induce the perception of a linear acceleration.

Tilt-coordination technique is commonly used for simulations of "heading" (term introduced by Gibson in 1950 [17] to describe linear self-motion along the anterior-posterior axis). A good example is the study of Groen et al. [19], which showed that a visuo-vestibular simulation of linear acceleration is perceived as being more realistic than a pure visual simulation. Using a balancing chair and a flat screen attached to the chair, they rotated the participants around the sagittal axis, presenting them with a sinusoidal forward-backward visual motion, synchronized with the inclination of the chair. By varying the signal frequency (tilt amplitude and visual acceleration amplitude), they observed that rotational velocity is more effective than tilt amplitude in inducing the perception of linear self-motion. Moreover, the threshold of tilt amplitude and rotation velocity depended on the visual acceleration, reaching $12 \mathrm{deg}$ and $3.4 \mathrm{deg} / \mathrm{s}$ respectively, for a low frequency $(0.04 \mathrm{~Hz})$ and a visual acceleration of $1.76 \mathrm{~m} / \mathrm{s}^{2}$. However, the authors advised generally not exceeding a rotation velocity of $2-4 \mathrm{deg} / \mathrm{s}$. This range covers motions with a frequency between 0.04 and $0.33 \mathrm{~Hz}$, where the threshold values depend on the frequency of motion and visual acceleration [19]. Similarly, A.J. Benson defines the detection threshold of angular velocity determined in a series of experiments as $3.7 \mathrm{deg} / \mathrm{s}$ [2].

As heading is the main driving situation, most dynamic driving simulators use tilt-coordination, even though it allows accelerations to be simulated only at low rates, because of the thresholds of detection of rotational velocity by the semi-circular canals [19]. To extend the range of possible simulations, the "washout" algorithm also adds linear translations to tilt and visu-

\footnotetext{
${ }^{1}$ According to Einstein's Principle of Equivalence, a pure accelerometer cannot distinguish between the inertia induced by acceleration and the effect of gravity.
} 
ally induced vection. In this way, linear accelerations are simulated by both tilt and translation, the distribution between them depending on the frequency of the desired acceleration: low-frequency accelerations are simulated by tilt, while high-frequency accelerations are simulated by translations [31]. However, due to the lack of knowledge on multisensory integration, the tilt/translation ratio is currently chosen empirically. In addition, it has been shown that small translations do not significantly improve the perception of linear acceleration [3]. In their study, the authors used a 6 degrees-of-freedom hexapod to simulate both translations and inclinations. The range of linear motions was between 0 and $0.5 \mathrm{~m}$ and the rotation between 0 and $15 \mathrm{deg}$. They used a monocular visual display simulating a linear motion on a field with depth cues (objects and people placed randomly). Manipulating the acceleration-deceleration (maximal acceleration of $1.25 \mathrm{~m} / \mathrm{s}^{2}$ and maximal deceleration of $-0.31 \mathrm{~m} / \mathrm{s}^{2}$ ), mixed with forward-backward tilt of the platform, they tested which scenario was more realistic. Their results show that a correct-to-slightly-exaggerated body tilt is rated as most realistic, if accompanied by visual acceleration. The most striking result is that a short forwardbackward translation does not have a major influence on the perception of acceleration. This loss of influence could be due to the small range of translation allowed by the hexapod. In more sophisticated simulators, this limitation no longer applies, because of the presence of an X-Y platform allowing motions from $5 \mathrm{~m}$ to $40 \mathrm{~m}$ in both directions, forward-backward and lateral (for a review see $[10,32])$. With this type of simulator, the "washout" algorithm can be used by combining the tilt of the hexapod and linear translations of the X-Y platform.

However, the tilt/translation ratio has not been investigated and is still chosen empirically. It is not clear which ratio leads to the most accurate perception of acceleration. Given the studies of Holly and McCollum [22] about perceptually equivalent situations under certain conditions, a change in tilt/translation ratio should not interfere with the perception of deceleration during a simulated braking situation, especially if all other sensorial cues are constant (visual and auditory cues). However, the conditions in which the vestibular system is stimulated in a driving simulator differ notably from the conditions in the theoretical study of Holly and McCollum [22] (in a simulator, the stimulations are not constant and never in complete dark). Therefore, the aim of our study was to verify whether the perception of a certain value of deceleration remains the same regardless of tilt/translation ratio, or whether it changes with this ratio.

\section{General methods}

\subsection{Participants}

Fourteen volunteers ( 3 women and 11 men), aged between 21 and 38 (mean 27.28), with normal or corrected-to-normal vision, participated in this study. They all had between 5 and 18 years (mean 9) driving experience, with an average frequency of 3.7 days/week and no or very little driving simulation experience. All subjects gave informed consent in compliance with the requirements of the ethical committee which governs and regulates human experimentation in France.

\subsection{Experimental device: The driving simulator}

The device used for this experiment was a dynamic driving simulator, composed of three main parts: a honeycomb structure or cell, a hexapod and an X-Y motion platform (Fig. 1). The cell contains a half-cab Citroen $\mathrm{C} 1$ fully-equipped (2 front adjustable seats, seat belts, steering wheel, pedals, gearbox, rearview mirror and side-view mirrors) where the driver sits. The hexapod makes it possible to move the cell along the $6 \mathrm{deg}$.-offreedom, the rotating point being the H-point (the hip of the driver). The translational movements are limited to $\pm 5 \mathrm{~m}, \pm 2.75 \mathrm{~m}$ and $\pm 20 \mathrm{~cm}$, on $X$, Y and $\mathrm{Z}$ respectively. The rotational movements are limited to $\pm 18 \mathrm{deg}, \pm 18 \mathrm{deg}$ and \pm 23 degrees, on pitch, roll and yaw respectively (see [10] for more details). The X-Y motion platform can reproduce linear movements of 10 and 5 meters. The maximum acceleration is $5 \mathrm{~m} / \mathrm{s}^{2}$. This is, of course, limited in time because of the "mechanical" braking of the simulator (each time the simulator accelerates it must brake before reaching the end stop). Therefore, both acceleration and deceleration have to be fitted into the same distance range (e.g. $10 \mathrm{~m}$ in the longitudinal direction).

The cell is equipped with a sound restitution system composed of 6 loudspeakers and a subwoofer. It is also surrounded by 3 flat screens which give a $160^{\circ}$ (horizontal) by $25^{\circ}$ (vertical) field of view. The projection is binocular and monoscopic, adjusted to the driver's field of vision (right-hand traffic). The rear-view mirrors are replaced by small screens that visually simulate a realistic rear view. During our study, these mirrors were not used (to avoid the subject self-positioning in the space, which could have facilitated the task and distorted the results). The visual scenarios were projected through three projectors, but the field of view was reduced to $120^{\circ}$ wide by covering part of the lateral windows. This was necessary because of the cell openings (Fig. 1B), which could have made the motion of the simulator relative to the room visible. 


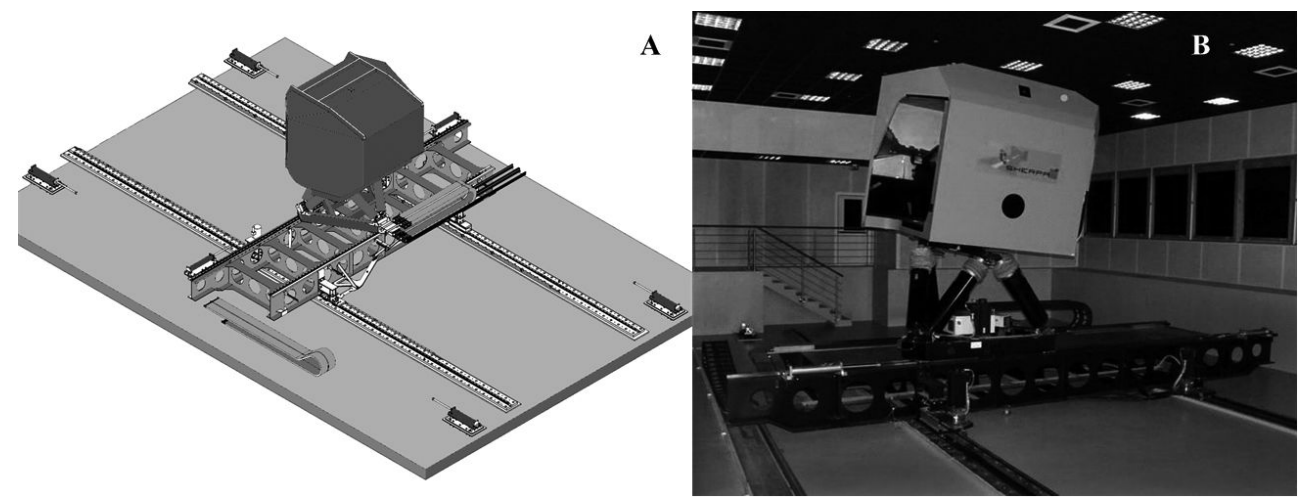

Fig. 1. Dynamic driving simulator SHERPA by PSA Peugeot-Citroën. The simulator is equipped with a 6 degrees-of-freedom hexapod and an X-Y motion platform of 10 by 5 meters (A). The structure placed on the hexapod (B) contains a half-cab of a Citroën C1, fully equipped. The visual scenes are projected on 3 flat screens placed in front of the vehicle cab in a semi-hexagon configuration, allowing a field-of-view 160 degrees wide and 25 degrees high.

\subsection{Stimuli}

Participants were exposed to different scenarios containing visual and dynamic stimulations, and representing the braking of a car after exposure to constant velocity.

The visual stimulation consisted of a one-lane road (3.6 $\mathrm{m}$ wide, including the white borders) surrounded by an empty green field. The road was composed of two consecutive parts: the first part, $200 \mathrm{~m}$ long, from the starting point to a white line drawn on the pavement, was bordered by trees at $18 \mathrm{~m}$ intervals (Fig. 2A). The second part of the road began from the white line and ended at a red wall, placed transversally on the pavement $23 \mathrm{~m}$ after the white line (Fig. 2B). The dimensions of the wall were: $3 \mathrm{~m}$ wide, $2.5 \mathrm{~m}$ high and $0.1 \mathrm{~m}$ deep. To avoid spatial positioning, that is to avoid the participants detecting the position of the wall relative to the objects placed in the vicinity of the road, no granular texture was used on the road or on the field (they were both of uniform color). For the same reason, there were no trees bordering the second part of the road, but there were a number of trees placed at the horizon line in the central visual field of the participant, at a distance of 400-900 $\mathrm{m}$ from the starting point, meaning 200-700 $\mathrm{m}$ from the white line (Fig. 2C). They were placed this far away in order to prevent the participants from using them as landmarks.

The dynamic stimulation, controlling the motion of the cell, consisted in simulating braking through tilt and translation. We used five different ratios of tilt and translation to simulate the same final deceleration, following a cosine curve with a peak of $-0.8 \mathrm{~m} / \mathrm{s}^{2}$ (Fig. 3 ). The ratios were composed of inverse-proportional per-
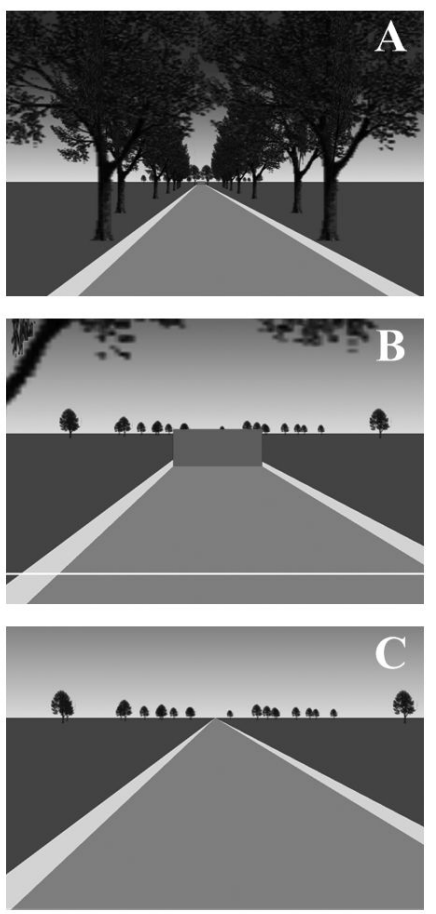

Fig. 2. The visual scene viewed at different points on the trajectory of the car: $\mathrm{A}$ - the visual scene viewed from the starting point; $\mathrm{B}$ the visual scene viewed just before the car crosses the white line; $\mathrm{C}$ the visual scene viewed during braking, after crossing the white line and after the wall has disappeared. During the latter, the subject is able to see the trees placed at the horizon line.

centages of tilt and translation. In condition 1, deceleration was simulated by pure tilt ( $100 \%$ tilt) and no translation was used ( $0 \%$ translation). The rotation acceleration of the cell followed the curve in Fig. 3A. In conditions 2, 3 and 4, translation was added gradually, 

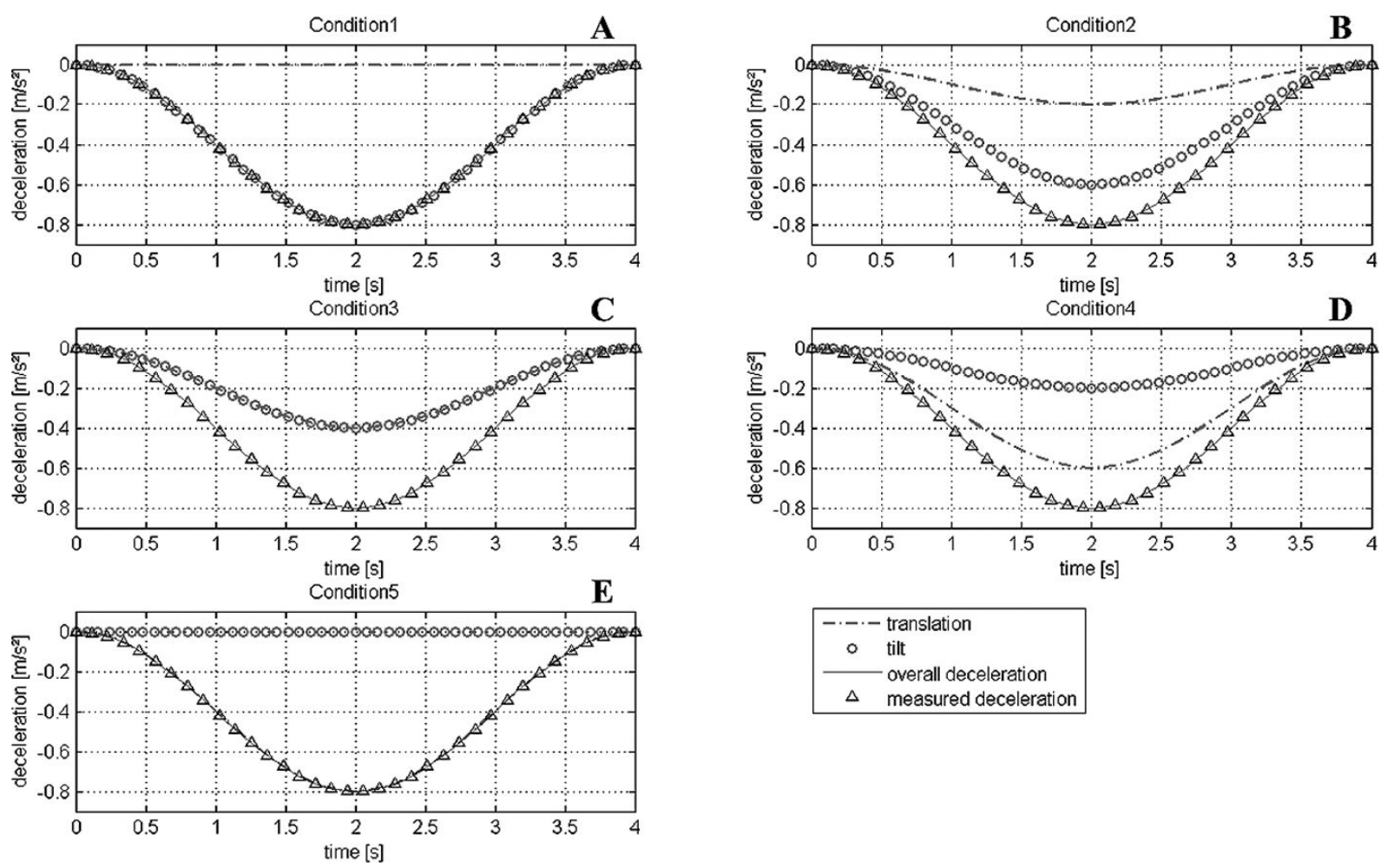

Fig. 3. Sinusoidal profiles of deceleration simulated by translation (dash-dot line) and of equivalent deceleration simulated by tilt (circles), represented for each of five conditions. The sum of the two signals is always the same, with a peak of $0.8 \mathrm{~m} / \mathrm{s}^{2}$ (solid line). The latter is compared to the signals measured by the three-dimensional accelerometer (triangles). The graphics clearly show that these two signals are identical and constant through the conditions. The two first signals, translation and tilt, are varying depending on condition. (A) Condition $1-$ the deceleration is represented only by tilt with a peak of $0.8 \mathrm{~m} / \mathrm{s}^{2}$ (circles). (B) Condition $2-$ the deceleration is represented by tilt with a peak of $0.6 \mathrm{~m} / \mathrm{s}^{2}$ (circles) and translation with a peak of $0.2 \mathrm{~m} / \mathrm{s}^{2}$ (dash-dot line). (C) Condition 3 - the deceleration is represented by equal tilt and translation, both with a peak of $0.4 \mathrm{~m} / \mathrm{s}^{2}$ (circles and dash-dot line overlaid). (D) Condition $4-$ the deceleration is represented by tilt with a peak of $0.2 \mathrm{~m} / \mathrm{s}^{2}$ (circles) and translation with a peak of $0.6 \mathrm{~m} / \mathrm{s}^{2}$ (dash-dot line). (E) Condition 5 - the deceleration is represented only by translation, with a peak of $0.8 \mathrm{~m} / \mathrm{s}^{2}$ (dash-dot line).

going from $25 \%$ to $50 \%$ and finally $75 \%$, while the ratio of tilt decreased from $75 \%$ to $50 \%$ and finally to $25 \%$ (Figs 3B, 3C and 3D). In the last condition, deceleration was simulated by pure translation which also followed the same curve as in condition 1 (Fig. 3E). All these conditions are presented in Table 1.

For all experimental conditions, the rotation velocities and tilt angles were kept under the thresholds of detection of vestibular system $(3.7 \mathrm{deg} / \mathrm{s}[2,19]$ for rotation velocity and $6 \mathrm{deg}$ [8] for inclination).

In addition, we used a three-dimensional accelerometer placed inside the cell to record the real motion of the cell and to verify that the desired deceleration level was achieved. The output signals were always the same across all conditions, confirming that, in terms of overall deceleration, all conditions were strictly equivalent. Accelerometer readings are presented in Fig. 3.

In order to determine the effects of visual cues, another condition (condition zero) of pure visual stim- ulation of the braking (no dynamic stimulation) was added.

\subsection{Task}

The participants were seated in the driver's seat, but had no access to controls during the simulation (passive simulation). They were asked to remain as far as possible in the same position, without touching the steering wheel or the pedals, head resting on the headrest and seat-belt fastened (a three-point seatbelt maintained the upper body immobile). They were also asked to wear earplugs to filter some of the noise made by the hexapod and the X-Y motion platform, to prevent the participants from guessing the position of the simulator in the room.

The session started with the car stationary at the starting point. Then, the car drove off towards the red 
Table 1

Description of conditions during the test for dynamic stimulation. The overall deceleration remained constant during conditions $1-5\left(0.8 \mathrm{~m} / \mathrm{s}^{2}\right)$, calculated as the sum of the deceleration simulated by translation and the equivalent deceleration simulated by tilt. The tilt is also described in terms of maximal inclination and rotational velocity corresponding to each deceleration. For condition 0 there is no motion, only visual simulation, and therefore the overall acceleration is also 0

\begin{tabular}{cccccc}
\hline & $\begin{array}{c}\text { Overall } \\
\text { deceleration }\end{array}$ & Translation & & Tilt & \\
\cline { 3 - 6 } Condition & $\mathrm{m} / \mathrm{s}^{2}$ & $\mathrm{~m} / \mathrm{s}^{2}$ & $\mathrm{~m} / \mathrm{s}^{2}$ & $\mathrm{deg}$ & $\mathrm{deg} / \mathrm{s}$ \\
\cline { 2 - 6 } & 0 & 0 & 0 & 0 & 0 \\
\hline 0 & 0.8 & 0 & 0.80 & 4.7 & 3.673 \\
1 & 0.8 & 0.2 & 0.60 & 3.5 & 2.754 \\
2 & 0.8 & 0.4 & 0.40 & 2.3 & 1.835 \\
3 & 0.8 & 0.6 & 0.20 & 1.2 & 0.917 \\
4 & 0.8 & 0.8 & 0.00 & 0 & 0 \\
5 & & & & & $\begin{array}{c}\text { Maximal } \\
\text { inclination }\end{array}$ \\
\hline
\end{tabular}

wall, accelerating for about 6 seconds, before reaching a constant speed of $50 \mathrm{~km} / \mathrm{h}$. After 20 seconds at constant speed, it crossed the white line and started to brake until it came to a stop. At the moment the car started to brake (as it crossed the white line), the red wall instantly disappeared (Fig. 2C). The braking lasted 4 seconds before the car came to a full stop. Because the distance between the white line and the wall and the duration of braking were held constant across trials, the car always stopped in the same position relative to the wall, which is with its bumper gently touching the wall. However, subjects were not informed about this invariability. We used a two-alternative-forced-choice paradigm (2AFC) to ask the subjects to evaluate the intensity of the braking. Thus, once the car had come to a full stop, the participants were asked to answer the following question "Did the car stop BEFORE or AFTER the red wall?". Participants had to answer this question very rapidly, once the car had stopped. They were also asked to rate the certainty of their answer from 1 to 6 , with 1 representing the lowest certainty, and 6 representing the highest certainty.

\subsection{Protocol}

The experiment started with a familiarization period in order to allow the participants to get used to the virtual environment of the simulator and to become familiar with the dimensions of the car. For this reason, the participants were exposed to a similar visual scenario without simulator motion (static simulation). The car drove towards to red wall, at a constant speed of $90 \mathrm{~km} / \mathrm{h}$. It started to brake the moment it crossed the white line, but this time the wall did not disappear and the car stopped in front of it, touching it with its bumper. The distances between the white line and the wall were varied for the familiarization session and were never identical with the distance used in the experimental session $(27 \mathrm{~m}, 37 \mathrm{~m}, 71 \mathrm{~m}$ and $59 \mathrm{~m})$. The durations of braking were also changed for the training period $(2 \mathrm{~s}, 3 \mathrm{~s}, 5 \mathrm{~s}$ and $6 \mathrm{~s}$ respectively). These 4 cases were presented randomly during a series of 8 trials $(2$ trials per case).

Once the familiarization period was completed, the experimental session started. The 6 conditions presented in Table 1 were repeated 6 times, for a total of 36 trials, ordered semi-randomly (no condition was ever presented twice consecutively). To avoid fatigue or lassitude, we organized the session as 4 series of 9 trials, with subjects allowed to take a break if they wished between series.

\subsection{Data analysis}

The performance of the task was analyzed in terms of braking perception errors. In order to perform these analyses, a score of -1 was attributed to any BEFORE answer, and a score of 1 to any AFTER answer, and these scores were multiplied by the corresponding level of certainty. The median of braking evaluation was calculated for each participant for each condition and represented graphically. Because of condition 0 , the configuration of the parameters was not square. As a result, this particular condition was analyzed separately. For all other conditions, a repeated-measures analysis of variance (ANOVA) and a post-doc Duncan test were conducted. The results of ANOVA showed that the perception of braking was non-linear. For this reason, a psychometric analysis was also conducted, where each BEFORE answer was equated with a score 


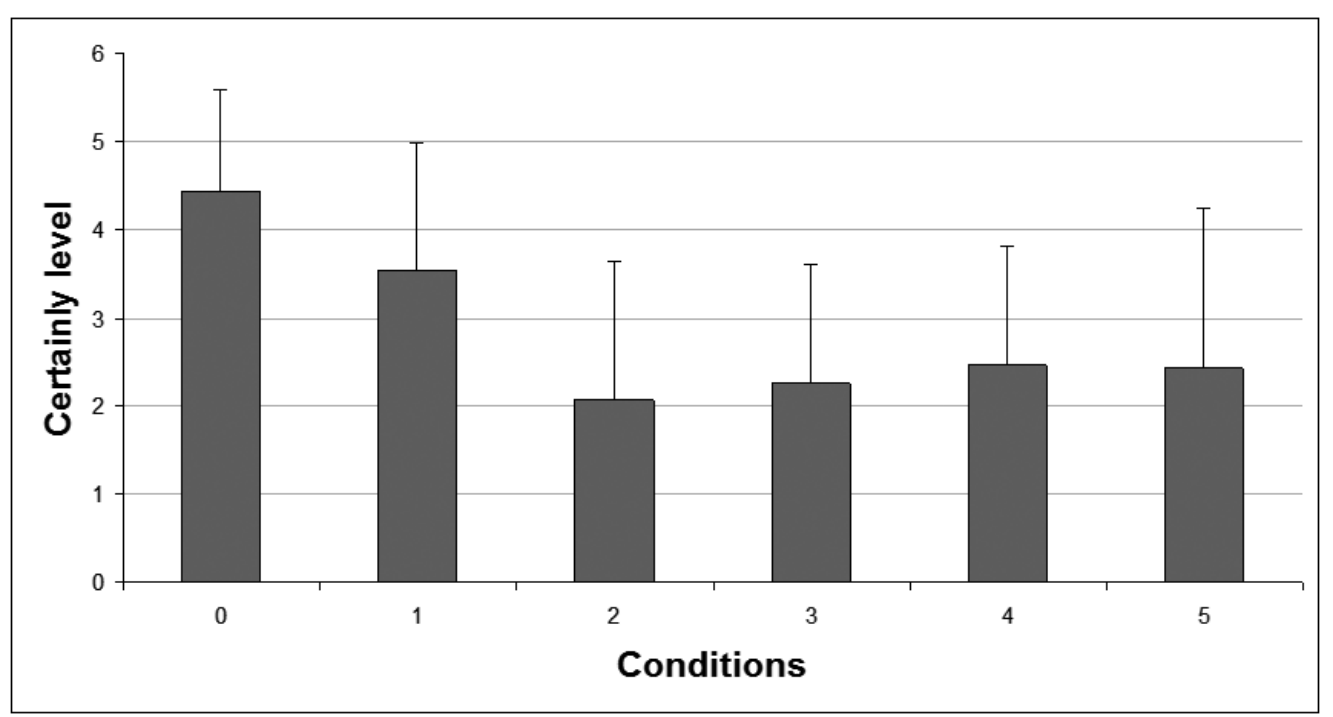

Fig. 4. Mean values of certainty levels. The maximum value (6) represents a $100 \%$ certainty, while the minimum value (1) represents a $0 \%$ certainty.

of 0 (if braking is strong enough, the probability of stopping after the wall is 0), and each AFTER answer was equated with a score of 1 (if braking is too weak, the probability of stopping after the wall is 1). The psychometric function was computed to determine the tilt/translation ratio for which the probability $p$ of perceiving the car before the wall is 0.5 . We used a Probit model for non-linear regression, which is suitable for forced-choice methods (binary type of responses). The function can be described as:

$$
P S E=a+(b-a) * \frac{0.5-p_{a}}{p_{b}-p_{a}}
$$

where $i$ is the condition number, $j$ the trial number, $P_{i}$ represents the probability that condition $i$ will elicit an AFTER answer, $C_{i, j}$ is the response for condition $i$ and trial $j, C_{0}$ is the condition for which probability $p=$ 0.5 and $n$ represents the power of the effect.

\section{Results}

Even though the participants detected differences between trials during braking, they were never aware of the different ratios of tilt/translation used to simulate the deceleration. Moreover, none of the participants realized that the wall was always in the same place. Nevertheless, they considered the task difficult, especially in the condition without dynamic stimulation, when they failed to detect that the car had come to a stop.

\subsection{Level of certainty}

The level of certainty describes the degree of faith participants had in their perception. In fact, the most realistic perceptions were coupled with the lowest certainty level, because the participants felt the car was so close to the wall that they could not detect whether it had stopped before or after the wall. As shown in Fig. 4, the highest levels of certainty found during our experiment were for condition 0 (mean value: 4.43 ). For the other conditions, the level of certainty is quasi-constant (Fig. 4), with a mean value of 2.55 .

\subsection{Evaluation of braking perception}

The results presented in Fig. 5 show the number of BEFORE/AFTER responses for each condition. For conditions 0,4 and 5, most participants evaluated the braking as weak $(93 \%, 64 \%$ and $64 \%$ of AFTER answers), whereas for conditions 1,2 and 3, most of the participants evaluated the braking as strong (93\%, $71 \%$ and $71 \%$ of BEFORE answers). Differences between BEFORE answers are increasing from condition 1 (pure tilt) to condition 5(pure translation), while differences between AFTER answers are decreasing. However, condition 0 leads to completely different results: subjects reported the sensation that the car never came to a full stop. This condition was particularly reliable, as confirmed by the high level of certainty ( 4.43 out of 6). 


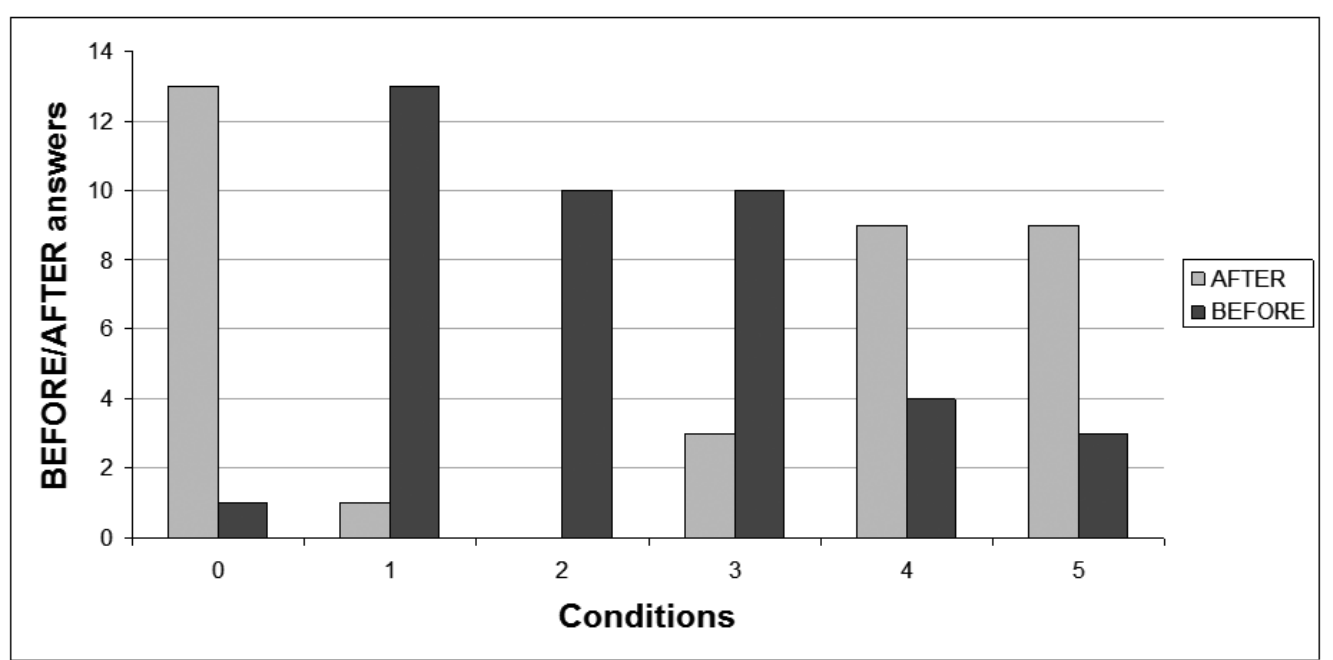

Fig. 5. The number participants answering BEFORE or AFTER for each condition. For each participant only one answer was retained (the median value of the 6 trials).

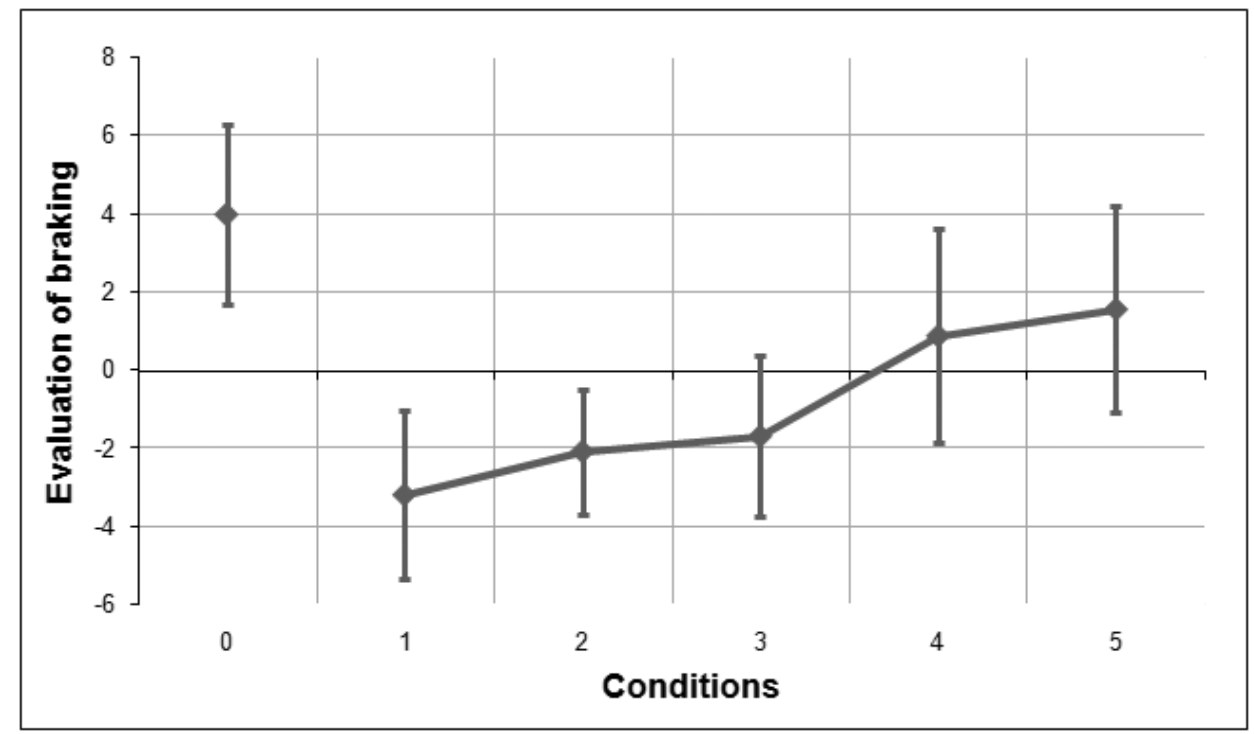

Fig. 6. Mean values of perception errors for each condition. The coefficient of perception error is obtained by multiplying two variables: 1 . the mean certainty level attributed by the participant; 2. a value of -1 for a BEFORE response or a value of 1 for an AFTER response. Negative values for perception error are equivalent to an overestimation of the braking (participants considered the car stopping before the wall). Positive values are equivalent to an underestimation of the braking (participants considered the car stopping after the wall).

Figure 6 presents the mean values of perception error for all conditions. This error ranges from a negative value for condition $1(-3.18)$ (pure tilt) to a positive value for condition 5 (1.57) (pure translation), while condition 0 stands out, showing a strong positive value (4). Negative values represent an overestimation of braking, while positive values represent an underestimation of braking. The point where there is no error (perception error $=0$ ) lies between conditions 3 and 4 .
Both Figs 5 and 6 show that deceleration is more weakly perceived when there is greater translation, more strongly perceived when there is greater tilt, and not perceived at all when there is no motion (in condition 0 ). This last result unsurprisingly suggests that absence of motion combined with lack of visual stimulation (no texture on the road and no bordering trees during the deceleration phase) is unfavorable to the perception of self-motion. In the light of these results, 
Table 2

The results of Duncan post-hoc test revealing a distribution pattern for the ratios. The test uses multiple comparisons between the mean values of each condition (values obtained by multiplying two variables: 1 . the certainty level attributed by participants; 2. a value of -1 for a BEFORE response or a value of 1 for an AFTER response). Pattern is represented by the signification values, by means of probability (for $p=1$, there is $0 \%$ probability that another ratio could fit the first group, while probabilities increase to $38 \%$ and $5 \%$ for the second and the third group respectively)

\begin{tabular}{cccccc}
\hline & & & \multicolumn{3}{c}{ Post-hoc Duncan test } \\
\cline { 3 - 6 } Condition & $\begin{array}{c}\text { Translation } \\
\mathrm{m} / \mathrm{s}^{2}\end{array}$ & $\begin{array}{c}\text { Tilt } \\
\mathrm{m} / \mathrm{s}^{2}\end{array}$ & 1st group & 2nd group & 3rd group \\
\hline 1 & 0.0 & 0.8 & -4.42 & & \\
2 & 0.2 & 0.6 & & -2.26 & \\
3 & 0.4 & 0.4 & & -1.84 & \\
4 & 0.6 & 0.2 & & & 2.00 \\
5 & 0.8 & 0.0 & & 0.620 & 0.950 \\
\hline
\end{tabular}

condition 0 is excluded from the rest of the analysis.

The ANOVA analysis for the five tilt/translation ratios shows a significant difference among these ratios ( $p=0.000, F=18.156)$. The post-hoc Duncan test classifies the five ratios into three groups (Table 2).

The first group concerns condition 1, where only tilt was used. The inclination of the cell was maximum 4.7 degrees, with a maximum rotational speed of $3.673 \mathrm{deg} / \mathrm{s}$. Even though these parameters remained below the threshold of perception [2,19], meaning that the tilt should not have been perceived, the simulated braking was perceived as quite strong. This perception was confirmed by most of the participants $(89,3 \%)$ and could have been induced by feeling the natural tilt in the car during the braking (there was no visual tilt simulated during the test). The percentage of subjects perceiving strong braking decreased in the second group of conditions (second and the third conditions), both with about $70 \%$ of BEFORE answers $(78.6 \%$ and $71.4 \%$ respectively), and even more for the third group (fourth and the fifth conditions), both with low percentages of BEFORE answers (35.7\% and $25 \%$ respectively).

In view of these differences among conditions, a psychometric function was computed to determine which of the ratios of tilt/translation is perceived as the most realistic (Fig. 7). The function can be described as:

$$
y=\operatorname{inormal}(-1.8438+(0.508525) * x, 0,1)
$$

The threshold of perception between BEFORE/ AFTER is defined by the value deduced from $50 \%$ AFTER responses. In our case, the threshold or the point of subjective equality (PSE) lies between condition 3 and condition 4 (Fig. 7), being PSE = 3.6. Given that the ratios of tilt and translation of each condition represent a percentage of $0.8 \mathrm{~m} / \mathrm{s}^{2}$, where the ratio of tilt is inversely proportional to the ratio of translation, we computed the percentage equivalent for a condition 3.6. Therefore, the PSE corresponds for a ratio of $35 / 65 \%$ tilt/translation, meaning $0.28 \mathrm{~m} / \mathrm{s}^{2}$ in tilt and $0.52 \mathrm{~m} / \mathrm{s}^{2}$ in translation. For this case, the corresponding angle of inclination is 1.64 degrees, while the rotation velocity is $1.284 \mathrm{deg} / \mathrm{s}$.

\section{Discussion}

The aim of the present study was to test whether changing tilt/translation ratio during a simulated deceleration influences the final perception of braking. We find that the perception does not remain constant across these changes, but rather that it depends on the proportion of tilt and translation. With a high proportion of tilt, braking is perceived as stronger than when more translation is used. However, we know that these ratios are equivalent from a physical point of view, because the real linear decelerations were recorded by a three-dimensional accelerometer placed inside the cell, which issued identical signals throughout the test, regardless of tilt/translation ratio. Thus the different perceptions induced by the different ratios are of some importance, since they cannot be ascribed to a difference in the deceleration stimulus. An explanation should probably be sought in the functionality of the vestibular system and the interactions among all the sensorial cues involved in the process of multi-sensory integration during the braking.

Two questions arise from our findings: 1) Why do different ratios not produce the same perception? and 2) Which ratio leads to the perception of deceleration that is closest to the real deceleration? 


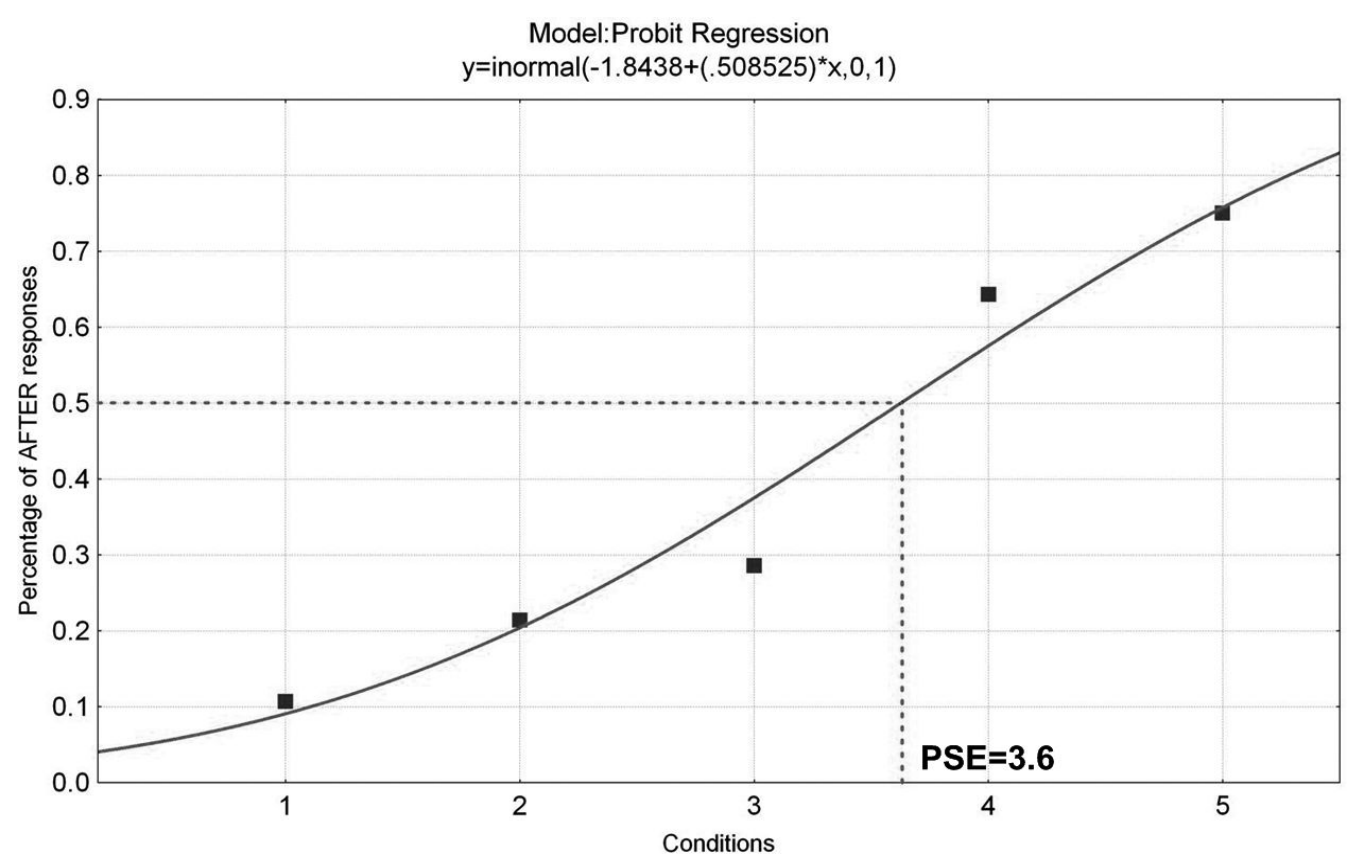

Fig. 7. Probit regression calculated for conditions 1 to 5 . The point of subjective equality (PSE) corresponds to the condition for which participants answered AFTER in 50\% of the case and BEFORE for the other 50\%. In this study, the PSE value is 3.6.

\subsection{What is a realistic tilt/translation ratio?}

As shown by the psychometric computations, the ratio responsible for the most realistic perception of deceleration is neither pure tilt nor pure translation, but a ratio of $35 \%$ tilt and $65 \%$ translation, corresponding to $0.28 \mathrm{~m} / \mathrm{s}^{2}$ in tilt and $0.52 \mathrm{~m} / \mathrm{s}^{2}$ in translation respectively. Therefore, while pure tilt combined with visual information improves the perception of braking compared to pure visual simulation [19], it seems that in our situation braking intensity is overestimated. In the same way, for pure translation, braking is underestimated.

Concerning the visual information, even though during the braking the only visual landmarks were the trees placed on the horizon line in the central visual field, the results show that this visual information had little influence on the final perception of the deceleration (condition 0 ). This lack of influence could be explained by the large distances between the white line and the trees on the horizontal line (between 200 and 700 meters). Being placed so far away, the angle of expansion of the trees was too small (about 0.2 degrees for the closest ones) so that the velocity derived from optic flow was perceived as non-existent. Various studies reveal that humans judge distances traveled on the basis of the velocity derived from optical flow $[7,16,28]$, which was almost non-existent in our case. Thus, the distance between the participants' eyes and the trees could have been perceived as constant throughout the test, leading to a perception of no motion, induced by these visual cues. Moreover, there were no landmarks in the peripheral field during the braking phase that could have produced lateral optic flow, which is known to provide information about the speed of motion [6]. As a result, there were no visual cues to describe the forward motion, and the only information available for the heading was non-visual cues.

According to Holly and McCollum [22], in static conditions both motion situations, tilt and translational acceleration, can be considered perceptually equivalent in the dark, making it impossible for an observer to distinguish between them. This is not the case in our study, since the visual scene is only compatible with a linear forward translation. Both cell tilt and cell translation should be interpreted in the same way. But our findings appear to invalidate this equivalence. Our results indicate that different tilt/translation ratios may be perceived differently. It seems that even when degrees of inclination and rotational velocities remain below the threshold of perception in the dark, the interaction between vestibular and non-vestibular cues produces different perceptions of braking, depending on the tilt/translation ratio. 


\subsection{The influence of vestibular cues}

While the bodies of the participants were maintained by three-point seatbelts, their heads were not immobilized. They were only instructed to keep their heads resting on the headrest. It is therefore possible that inclinations of the head due to braking varied from one ratio to another, depending on the amplitude and the velocity of the tilt of the cell and on whether or not the participants moved their heads. This should explain the differences in perception for the different ratios. Indeed, even though the level of rotational velocity of the cell was kept below the threshold of perception of semi-circular canals, we cannot exclude the possibility that the participants might have moved their heads during braking. This head motion could have been added to the tilt of the cell, increasing the level of vestibular stimulation and/or generating a proprioceptive stimulation at neck level. Therefore for condition 1 , where the rotational velocity of the cell $(3.67 \mathrm{deg} / \mathrm{s})$ was close to the threshold of perception defined by Benson (3.7 deg/s [2]) but still in the range described by Groen et al. (2-4 deg/s [19]), this threshold might have been exceeded by the addition of head motion. This would explain the statistical differences among the ratios, especially the perception of stronger braking for condition 1 . In the same way, with the second and third ratios, both falling within the second group, head rotational velocity $(2.75 \mathrm{deg} / \mathrm{s}$ and $1.83 \mathrm{deg} / \mathrm{s}$ respectively), might have been increased to nearer the limits (2-4 deg/s) determined by Groen et al. [19] but still below the perception threshold defined by Benson [2]. For the third group, we used only very weak rotational velocities (below $1 \mathrm{deg} / \mathrm{s}$ ), so the probability of perceiving the rotations is almost zero. Therefore, the weight of vestibular cues might have been increased by the motion of the head, dependent on the quantity of tilt (degree of inclination and angular velocity).

\subsection{Influence of non-vestibular cues}

Of course, in driving tasks, non-vestibular cues are also encountered and contribute to the sense of displacement. In particular, visual cues are considered to be highly relevant. But in our case the visual stimulation was kept intentionally scarce and constant throughout all conditions in order to reinforce the non-visual information. Therefore, visual information had no influence on braking perception, as borne out by results from condition 0 , where visual information alone was available (lack of dynamic motion): participants had the impression that the car did not stop. Nevertheless, even excluding visual cues, we are left with the possibility that the differences in perception for the different ratios were due to proprioceptive cues. Indeed, some authors $[8,33]$ have shown that proprioceptive cues can be the predominant information in the control and perception of postural orientation in quasi-static orientation, while detection of vestibular cues requires a greater postural change. During driving, differing pressure of the belt or of the backrest of the seat can provide some proprioceptive information about the motion of the body (and of the cell). But in our case, the pressure of the belt or the backrest was constant, since the inertial stimulation was maintained constant throughout the test, as measured by the three-dimensional accelerometer (see Fig. 3). Consequently, the only proprioceptive information available during braking was head motion, which may have influenced braking perception both directly (neck proprioception) and indirectly (via vestibular cues).

\section{Conclusion}

Using tilt-coordination together with translation appears to be a good method for simulating braking on a dynamic simulator. However, the combination of tilt and translation has to be precisely controlled to produce the expected perception and, for still undetermined reasons, a specific tilt/translation ratio must be used to simulate a given level of deceleration. Further studies should examine the validity of the specific ratio found here for different values of braking. As yet, it is impossible to conclude on whether this ratio remains constant over different levels of braking. Viewed from the perspective of the Bayesian approach [26,27,34], the contribution of each sensorial cue carries a certain weight, which represents a probability density function conditioned by "a priori" information. Here, this "a priori" may have been represented by the velocity of motion before the white line or the braking distance from the line to the wall. As, in this study, both are constant, they do not cause the variations observed in the perception of braking. But further studies, using different values of deceleration, as well as different velocities and braking distances, should yield more information about the relative weighting of these cues. This would allow us to develop a mathematical model explaining the perception of acceleration simulated by tilt and translation, leading to improved realism in driving simulators. 


\section{Acknowledgements}

The authors would like to thank Marjorie Sweetko for the language editing of this paper. This research was supported by a PhD grant from ANRT (Association Nationale de la Recherche et de la Technologie) and PSA Peugeot-Citroën. We also wish to thank the two anonymous reviewers for their helpful comments which allowed us to improve the quality of the manuscript.

\section{References}

[1] D.E. Angelaki, M. Wei and D.M. Merfeld, Vestibular discrimination of gravity and translational acceleration, Annals of the New York Academy of Sciences 942 (2001), 114-127.

[2] A.J. Benson, Sensory functions and limitations of the vestibular systems, in: Perception and Control of Self Motion, R. Warren and A.H. Wertheim, eds, Laurence Erlbaum Associates, Hinsdale, New Jersey, 1990, pp. 145-170.

[3] D.R. Berger, J. Schulte-Pelkum and H.H. Bülthoff, Simulating believable forward accelerations on a stewart motion platform, Technical report, Max Planck Institute for Biological Cybernetics, 2007.

[4] A. Berthoz, B. Pavard and L.R. Young, Perception of linear horizontal self-motion induced by peripheral vision (linear vection), Experimental Brain Research 23 (1965), 471-489.

[5] A. Berthoz and I. Viaud-Delmon, Multisensory integration in spatial orientation, Current Opinion in Neurobiology 9 (1999), 708-712.

[6] T. Brandt, J. Dichgans and E. Koenig, Differential effects of central versus peripheral vision on egocentric and exocentric motion perception, Experimental Brain Research 16 (1973), 476-491.

[7] F. Bremmer, M. Kubischik, M. Pekel, M. Lappe and K.P. Hoffmann, Linear vestibular self-motion signals in monkey medial superior temporal area, Annals of the New York Academy of Sciences 871 (1999), 272-281.

[8] L. Bringoux, V. Nougier, P.A. Barraud, L. Marin and C. Raphel, Contribution of somesthetic information to the perception of body orientation in the pitch dimension, The Quarterly Journal of Experimental Psychology 56 (2003), 909-923.

[9] L. Bringoux, S. Schmerber, V. Nougier, G. Dumas, P.A. Barraud and C. Raphel, Perception of slow pitch and roll body tilts in bilateral labyrinthine-defective subjects, Neuropsychologia 40 (2002), 367-372.

[10] T. Chapron and J.P. Colinot, The new PSA Peugeot-Citroën advanced driving simulator, Overall Design and Motion Cue Algorithm, in: Proceedings of Driving Simulation Conference, 2007.

[11] J. Dichgans and T. Brandt, Visual-vestibular interaction: Effects on self-motion perception and postural control, in: Handbook of Sensory Physiology, Perception, R. Held, H.W. Leibowitz and H.L. Teuber, eds, Springer, Berlin, 1978, pp. 755804.

[12] W.H. Ehrenstein and A. Ehrenstein, Psychophysical Methods, in: Modern Techniques in Neuroscience Research, U. Windhorst and H. Johansson, eds, Springer-Verlag Berlin Heidelberg, 1999, pp. 1211-1241.

[13] P. Feenstra, M. Wentink, B. Gracio Correia and W. Bles, Effect of simulator motion space on realism in the desdemona simulator, in: Proceedings of Driving Simulation Conference, 2009.

[14] N. Filliard, B. Vailleau, G. Reymond and A. Kemeny, Combined scale factors for lateral and yaw motion rendering to optimize driver's perception and control behavior, in: Proceedings of Driving Simulation Conference, 2009.

[15] R. Fitzpatrick and D.I. McCloskey, Proprioceptive, visual and vestibular thresholds for the perception of sway during standing in humans, Journal of Physiology 478 (1994), 173-186.

[16] H. Frenz, F. Bremmer and M. Lappe, Discrimination of travel distances from 'situated' optic flow, Vision Research 43 (2003), 2173-2183.

[17] J.J. Gibson, The Perception of the Visual World, Houghton Mifflin, Boston, 1950.

[18] J.J. Gibson, The Senses Considered as a Perceptual System, MIT Press, Cambridge, 1966.

[19] E.L. Groen and W. Bles, How to use body tilt for the simulation of linear self motion, Journal of Vestibular Research 14 (2004), 375-385.

[20] E.L. Groen, I.P. Howard and B.S. Cheung, Influence of body roll on visually induced sensations of self-tilt and rotation, Perception 28 (1999), 287-297.

[21] L.R. Harris, M. Jenkin and D.C. Zikovitz, Visual and nonvisual cues in the perception of linear self motion, Experimental Brain Research 135 (2000), 12-21.

[22] J.E. Holly and G. McCollum, The shape of self-motion perception - I. Equivalence classification for sustained motions, Neuroscience 70 (1996), 461-486.

[23] R.G. Kaptein and J.A. Van Gisbergen, Canal and otolith contributions to visual orientation constancy during sinusoidal roll rotation, Journal of Neurophysiology 95 (2006), 1936-1948.

[24] M.J. Kearns, W.H. Warren, A.P. Duchon and M.J. Tarr, Path integration from optic flow and body senses in a homing task, Perception 31 (2002), 349-374.

[25] A. Kemeny and F. Panerai, Evaluating perception in driving simulation experiments, Trends in Cognitive Sciences 7 (2003), 31-373.

[26] D.C. Knill and A. Pouget, The bayesian brain: the role of uncertainty in neural coding and computation, Trends in Neurosciences 27 (2004), 712-719.

[27] K.P. Kording and D.M. Wolpert, Bayesian decision theory in sensorimotor control, Trends in Cognitive Sciences 10 (2006), 319-326.

[28] D.N. Lee, The optic flow field: The foundation of vision, Philosophical Transactions of the Royal Society of London, Series B 290 (1980), 169-179.

[29] J.C. Lepecq, C. De Waele, S. Mertz-Josse, C. Teyssèdre, P.T. Huy, P.M. Baudonnière and P.P. Vidal, Galvanic vestibular stimulation modifies vection paths in healthy subjects, Journal of neurophysiology 95 (2006), 3199-3207.

[30] R. Loose, T. Probst and E.R. Wist, Perception of direction of visual motion. I. Influence of angular body acceleration and tilt, Behavioural Brain Research 81 (1996), 141-146.

[31] M.A. Nahon and L.D. Reid, Simulator motion-drive algorithm: A designer's perspective, Journal of Guidance, Control, and Dynamics 13 (1990), 356-362.

[32] M. Pinto, V. Cavallo and T. Ohlmann, The development of driving simulators: toward a multisensory solution, Le travail humain 71 (2008), 62-95.

[33] M. Vaugoyeau, S. Viel, B. Amblard, J.P. Azulay and C. Assaiante, Proprioceptive contribution of postural control as assessed from very slow oscillations of the support in healthy humans, Gait \& Posture 27 (2008), 294-302. 
[34] A. Yuille and H.H. Bülthoff, Bayesian decision theory and psychophysics, in: Perception as Bayesian Inference, D.C. Knill and W. Richards, eds, Cambridge University Press, New York, 1996, pp. 123-161.
[35] L.H. Zupan, D.M. Merfeld and C. Darlot, Using sensory weighting to model the influence of canal, otolith and visual cues on spatial orientation and eye movements, Biological Cybernetics 86 (2002), 209-230. 